\title{
Increased Expression of Y-Box-Binding Protein-1 in Hind-Limb Muscles During Regeneration from Ischemic Injury in Mice
}

\author{
Megumi Fuke, ${ }^{1}$ Makoto Narita, ${ }^{1}$ Yuko Wada, ${ }^{1}$ Tatsuichiro Seto, ${ }^{1}$ Kenji Okada, ${ }^{1}$ Jun \\ Nakayama, ${ }^{2}$ Hiroto Izumi $^{3}$ and Ken-ichi Ito ${ }^{1}$ \\ ${ }^{1}$ Department of Surgery (II), Shinshu University School of Medicine, Matsumoto, Nagano, Japan \\ ${ }^{2}$ Department of Molecular Pathology, Shinshu University Graduate School of Medicine, Matsumoto, Nagano, \\ Japan \\ ${ }^{3}$ Department of Occupational Pneumology, Institute of Industrial Ecological Sciences, University of Occupational \\ and Environmental Health, Kitakyushu, Fukuoka, Japan
}

\begin{abstract}
Critical limb ischemia (CLI) is the most severe complication of peripheral arterial disease (PAD). Understanding the molecular mechanisms underlying tissue repair after CLI is necessary for preventing PAD progression. Y-box binding protein-1 (YB-1) regulates the expression of many genes in response to environmental stresses. We aimed to determine whether YB-1 is involved in ischemic muscle regeneration. A mouse ischemic hind-limb model was generated; namely, the femoral, saphenous, and popliteal arteries in the left hind limb were ligated. The right hind limb, with skin incisions alone, served as control. Hind limbs ( $n=3-5$ for each time point) were examined on day 0 (before the operation) and on postoperative days $1,2,7,10$, and 14, and the biceps femoris, adductor, rectus femoris, and gracilis muscles were subjected to histopathological and immunohistochemical analyses. In ischemic limbs, myogenesis, triggered by an increase in myotubes, began on day 7; thereafter, regenerated muscles gradually increased in volume. RT-PCR analysis showed that YB-1 mRNA levels were increased in the limbs after ischemic injury, peaked on day 2, and subsequently decreased. On day 7 , expression levels of MyoD and alphasmooth muscle actin ( $\alpha S M A)$ mRNAs were significantly higher in ischemic muscles than in control muscles. Immunohistochemical analysis revealed increased YB-1 immunoreactivity in myoblasts and myotubes on day 7 , which was decreased by day 14 . The immunoreactive $\alpha \mathrm{SMA}$ and smooth muscle myosin heavy chain were transiently increased in myotubes. This is the first report showing the increased expression of YB-1 during muscle regeneration after ischemic injury.
\end{abstract}

Keywords: critical limb ischemia; ischemic injury; mouse model system; regeneration; YB-1

Tohoku J. Exp. Med., 2018 January, 244 (1), 53-62. (C) 2018 Tohoku University Medical Press

\section{Introduction}

Peripheral arterial disease (PAD) is a common atherosclerotic syndrome affecting more than 25 million individuals in Europe and the United States (McDermott et al. 2001; Belch et al. 2003; Norgren et al. 2007); moreover, its incidence has been increasing in Japan (Suzuki and Iso 2013). Critical limb ischemia (CLI) is the most severe complication of PAD, and approximately $1 \%$ of PAD patients develop CLI, which is associated with poor prognosis. To prevent PAD progression, pharmacotherapy to manage underlying diseases such as hypertension, diabetes, and dyslipidemia is prescribed, and therapy to improve blood flow with antiplatelet agents and vasodilators is administered (Weitz et al. 1996; Rowlands and Donnelly 2007). The prognosis of patients with PAD and those with CLI is unfavorable, and the 1-year mortality and major amputation rates are estimated to be approximately $25 \%$ and $30 \%$, respectively (Norgren et al. 2007). To date, surgical therapies such as bypass grafting or endarterectomy (or conventional revascularization therapies such as angioplasty, intravascular stents, or intra-arterial thrombolysis) are considered the most promising treatment options for ischemic limbs; however, these methods can be used to treat only approximately $5 \%$ of patients with PAD or CLI. Furthermore, persistent effects are observed in approximately $25 \%$ of patients after revascularization (Kawamoto et al. 2009). Hence, the development of a novel therapeutic strategy to improve the prognosis of patients with PAD and those with CLI is required.

The process of tissue repair after acute CLI is highly complicated and finely regulated. The restoration of ischemic tissue is a very complicated event wherein angiogenesis, myogenesis, and inflammation proceed simultaneously

Received August 18, 2017; revised and accepted December 25, 2017. Published online January 20, 2018; doi: 10.1620/tjem.244.53. Correspondence: Ken-ichi Ito, Division of Breast, Endocrine and Respiratory Surgery, Department of Surgery (II), Shinshu University School of Medicine, 3-1-1 Asahi, Matsumoto, Nagano 390-8621, Japan.

e-mail: kenito@shinshu-u.ac.jp 
and interact with one another. During ischemic injury repair, various cells can interact with one another; however, the molecular mechanisms underlying tissue repair are unclear.

Y-box binding protein-1 (YB-1) belongs to the highly conserved Y-box family of proteins (Wolffe et al. 1992). YB-1 was initially identified as a transcription factor that interacts with inverted CCAAT boxes (Y boxes) in eukaryotic promoters (Didier et al. 1988; Furukawa et al. 1998; Kohno et al. 2003). It is primarily localized in the cytoplasm but can be translocated to the nucleus in response to various environmental stresses. YB-1 regulates the transcription of genes involved in proliferation and differentiation, as well as chemotherapeutic resistance in cancer cells (Kohno et al. 2003; Fujita et al. 2005; Hyogotani et al. 2012; Ito et al. 2012).

Kamalov et al. (2005) reported that early YB-1 expression stimulates the proliferation and migration of myofibroblasts at the site of myocardial infarction in a rat model, suggesting that YB-1 upregulation promotes rapid bursts in cell proliferation during the early stages of healing. At the later stages of wound healing, YB-1 might be involved in extracellular matrix remodeling through the regulation of collagen and renin synthesis (Kamalov et al. 2005). Thus, numerous studies have suggested the possibility that YB-1 is involved in wound healing and tissue remodeling after ischemic injury. The present study aimed to determine whether YB-1 is involved in wound healing and remodeling of limb tissue after ischemic injury.

\section{Materials and Methods}

Animals

We used adult male BALB/c mice, aged 6-7 weeks (Japan Charles River Laboratories, Tokyo, Japan). The study protocol conformed to the Guidelines for the Handling of Animals of the Research Committee of Shinshu University and was approved by the Committee on the Ethics of Animal Experiments of the Shinshu University (Permit Number: 210057). The mice were anesthetized or euthanized through intraperitoneal injection of chloral hydrate, and all efforts were made to minimize animal suffering.

The mice were positioned in dorsal recumbency with their hind limbs externally rotated. A skin incision was made over the femoral artery beginning at the inguinal ligament and this continued caudally to the popliteal bifurcation. The femoral artery, saphenous artery, and popliteal artery in the left side hind limb of each mouse were isolated below the level of the popliteal bifurcation, and ligated using a 7-0 Prolene (Ethicon, Somerville, NJ, USA) suture. The right side hind limb, with skin incisions alone, was used as a control. Hind limbs (n $=3-5$ for each time point) were harvested for examination on day 0 (before the operation) and on postoperative days 1, 2, 7, 10, and 14 (Fig. 1). The biceps femoris, adductor, rectus femoris, and gracilis muscles in the harvested limbs were subjected to histopathological and immunohistochemical analyses.

\section{Histopathological and immunohistochemical analyses}

Hind limb tissues were fixed in $20 \%$ formalin, decalcified using K-CX (FALMA, Tokyo, Japan), embedded in paraffin, and cut into $3-\mu \mathrm{m}$-thick slices. The sections were deparaffinized with xylene and ethanol and subjected to hematoxylin and eosin staining using standard methods. After deparaffinization, the sections were irradiated in a microwave oven in $10 \mathrm{mmol} / \mathrm{L}$ citrate buffer ( $\mathrm{pH} 6.0$ ) for $15 \mathrm{~min}$

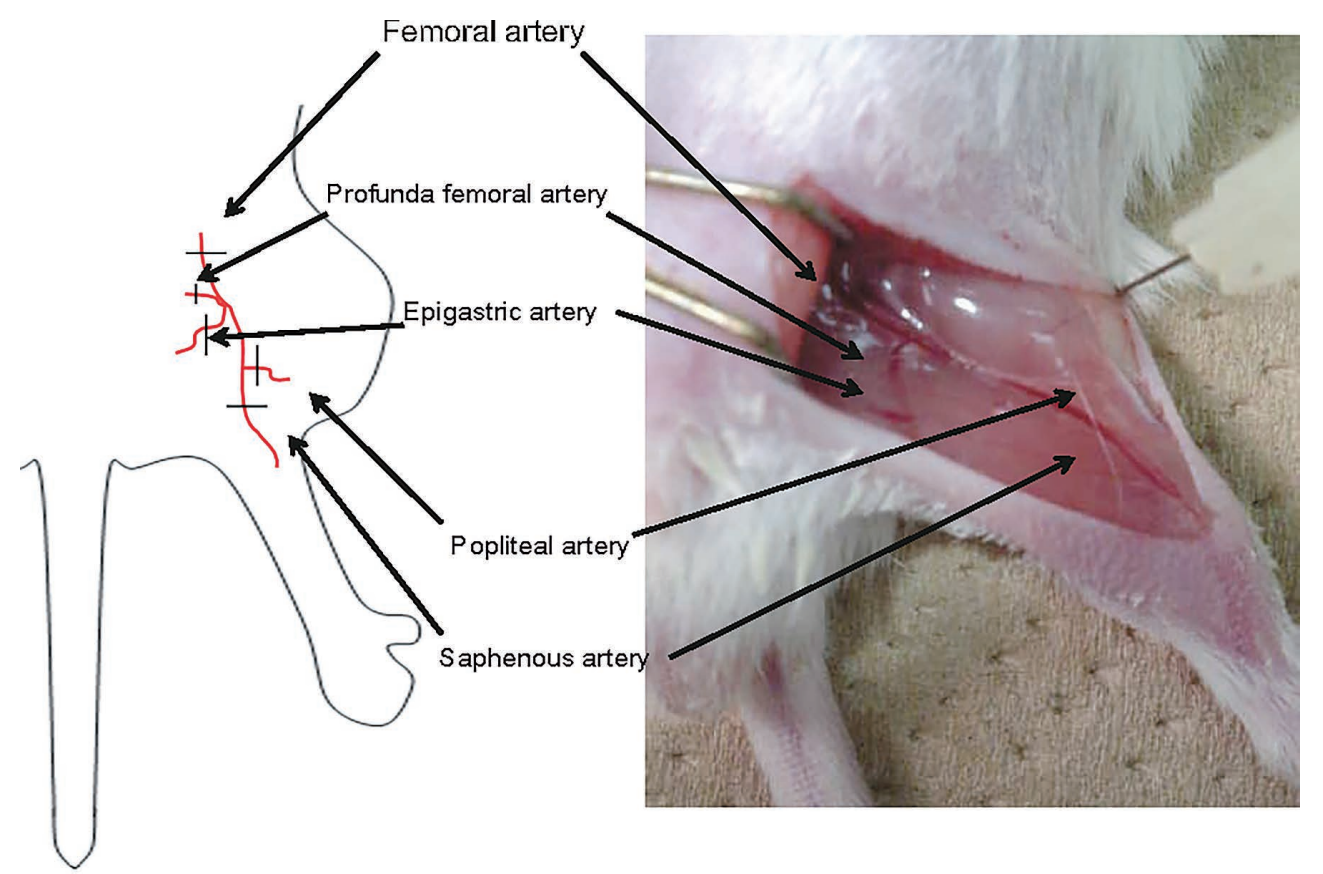

Fig. 1. Diagrammatic representation of mouse hind limb ischemic model.

The femoral artery, saphenous artery, and popliteal artery were isolated below the level of the popliteal bifurcation and ligated using a 7-0 Prolene suture. Hind limbs were harvested at day 0 (before the operation) and postoperative days 1 , $2,7,10$, and 14. Hind limbs with skin incisions alone were used as control. 
and pre-incubated with $0.3 \%$ hydrogen peroxide to eliminate nonspecific reactions. The sections were then incubated at $4{ }^{\circ} \mathrm{C}$ with rabbit-derived primary anti-CD31 antibody (Spring Bioscience, Pleasanton, CA, USA) or anti-YB-1 antibody (Abcam, Tokyo, Japan) for $24 \mathrm{~h}$ in a moist chamber. The sections were then incubated with biotinylated anti-rabbit antibody (Vector, Burlingame, CA) for 60 min. Antibody-biotin conjugate was detected using an $\mathrm{ABC}$ kit (Vector) in accordance with the manufacturer's instructions. For primary antibodies against MyoD (Abcam), alpha-smooth muscle actin ( $\alpha$ SMA) (Sigma, St. Louis, MO), and smooth muscle myosin heavy chain (SMemb/MHC-B; Abcam), we used the Vectastain MOM Immunodetection Kit (Vector) in accordance with the manufacturer's instructions.

\section{Real-time RT-PCR assay for mRNA quantification}

Hind limbs were rapidly dissected out and snap frozen in liquid nitrogen. Before homogenizing the frozen tissue, the skin and bone were removed from the specimen. Thereafter, total RNA was isolated from hind limb muscles, using the RNeasy Fibrous Tissue Mini Kit (Qiagen, Hilden, Germany) in accordance with the manufacturer's instructions. The relative mRNA levels for the genes of interest were assessed by RT-PCR using a 7300 Real-Time PCR System (Applied Biosystems, Foster City, CA, USA). TaqMan ${ }^{\circledR}$ Gene Expression Assays for mouse YB-1 (Mm00850878_g1), MyoD (Mm01203489_ g1), and $\alpha$ SMA (Mm01204962_gH) were purchased from Applied Biosystems (Carlsbad, CA, USA). mRNA levels were quantified in triplicate. The expression levels of interest mRNA were normalized to mRNA of glyceraldehyde 3-phosphate dehydrogenase (GAPDH) (\#4308313), using the $\Delta 4 \mathrm{Ct}$ comparative method.

\section{Statistical analysis}

Data are expressed as the mean \pm SD. Differences were compared using the Student's $t$-test. $P<0.05$ was considered significant.

\section{Results}

Regenerated muscle volume is increased via myogenesis on day 7

Histopathological findings were compared between hind limbs subjected to ischemic injury (Fig. 2A-F) and control limbs with skin incisions alone (Fig. 2G-L) after 14 days. The control group displayed no remarkable histopathological changes; however, the migration and infiltration of mononuclear inflammatory cells between muscular fibers were initially observed between days 1 and 2 in ischemic limbs. Thereafter, normal muscles decreased in volume, which was accompanied by an increase in adipose degeneration at day 7. Simultaneously, myogenesis, caused by an increase in myotube number, was initially observed on day 7 in ischemic limbs; subsequently, the regenerated muscles gradually increased in volume.

\section{MyoD, $\alpha S M A$, and YB-1 mRNA levels are increased tempo- rally in ischemic limbs}

We next tested alterations in YB-1, MyoD, and $\alpha \mathrm{SMA}$ mRNA levels in mouse limbs after ischemic injury by performing RT-PCR (Fig. 3). YB-1 mRNA levels were increased in the ischemic limbs immediately after injury, peaked on day 2 , and decreased temporally thereafter. The expression levels of MyoD and $\alpha$ SMA mRNAs in the ischemic limbs were peaked on day 7 after injury; they were significantly higher on day 7 in the ischemic limbs than in the control limbs $(p<0.05)$.

\section{$Y B-1, \alpha S M A, S M e m b$, and MyoD are expressed in the myo- tubes of ischemic hind limbs}

The expression of YB-1 and markers of endothelial cells and muscle differentiation were visualized through immunohistochemical staining to analyze microenvironmental changes that occur during regeneration after ischemic injury. One day after ischemic injury (day 1), YB-1 was localized mostly to histiocytes, macrophages, osteoblasts, and mononuclear inflammatory cells in the ischemic hind limbs and was not detected in the muscles until day 2. From day 7 to day 10, YB-1 levels increased temporally in the myoblasts and myotubes. Thereafter, YB-1 levels in the regenerated muscles decreased on day 14 (Fig. 4A-F).

CD31, an endothelial cell marker, was localized to the endothelial cells of the existing small arteries in the ischemic lesion alone until day 2 after injury. After day 7, CD31 levels increased in the regenerated lesion, which indicated the migration and proliferation of capillary endothelial cells to the injured area (Fig. 4G-L).

MyoD belongs to a family of myogenic regulatory factors and plays a key role in regulating muscle differentiation. It is considered one of the earliest markers of myogenic commitment and is expressed in the myosatellite cells, myoblasts, and myotubes. After ischemic injury, MyoD was localized to the myosatellite cells beneath the basal lamina of the myofiber alone until day 2. After day 7, MyoD levels increased in the newly generated myoblasts and myotubes (Fig. 5A-F).

aSMA, a marker of smooth muscle and myofibroblastic cells, was localized to the existing normal vascular smooth muscle cells until day 2. However, it was localized to myotubes on day 7, and its levels in the regenerated muscles were reduced on day 14 (Fig. 5G-L).

The embryonic form of SMemb/MHC-B, a marker of proliferating embryonic smooth muscle cells, was localized to the regenerated myotubes in the ischemic limbs in a manner similar to that of $\alpha \mathrm{SMA}$ (Fig. 6).

Upon analyzing serial sections of the ischemic limb on day 10, YB-1, $\alpha$ SMA, and SMemb were co-localized in the myotubes expressing MyoD (Fig. 7).

\section{Discussion}

This study reports the transient upregulation of YB-1 simultaneously with MyoD and $\alpha \mathrm{SMA}$ in the ischemic limbs of a murine model of hind limb ischemia. To our knowledge, this is the first study to report an alteration in YB-1 expression in ischemic limbs during the acute phase of injury. The present findings suggest that this transient upregulation of YB-1 is involved in the regeneration of skeletal muscles after ischemic injury. 
Ischemic limbs
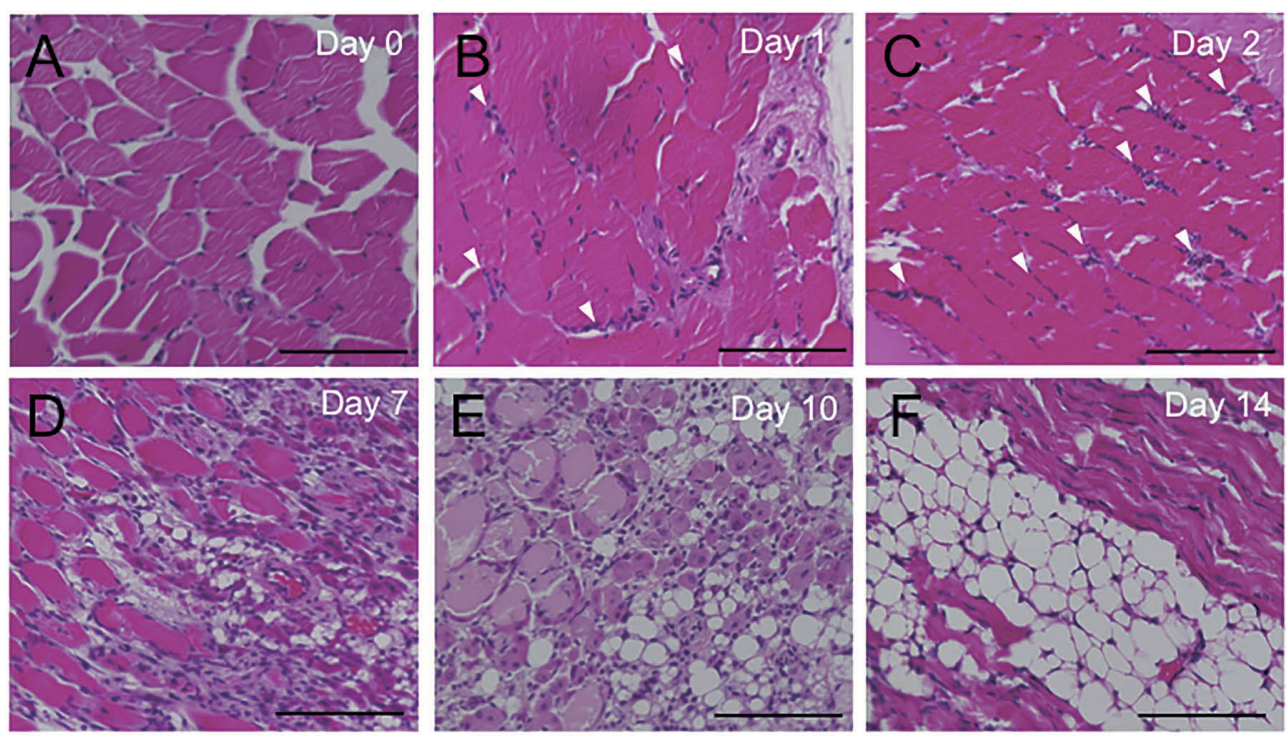

Control limbs
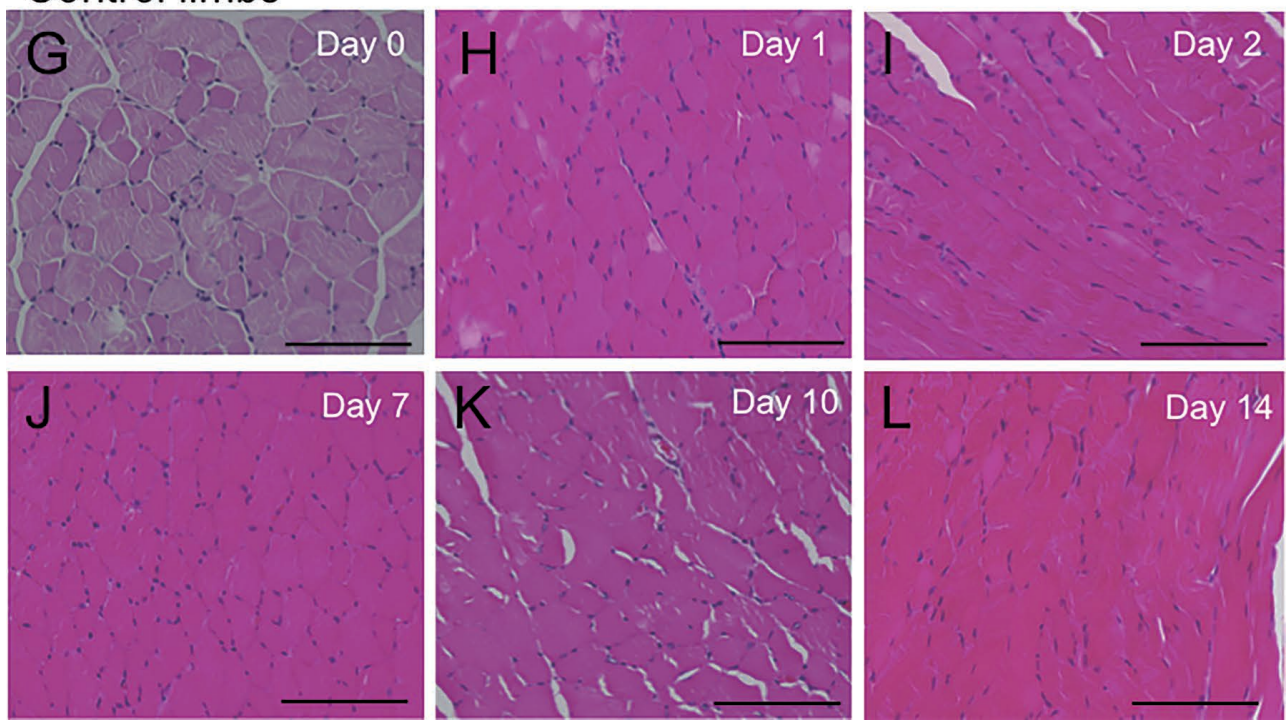

Fig. 2. Histopathological findings comparing the ischemic limbs to the control limbs.

(A-F) Hematoxylin-eosin (HE) staining of the ischemic limbs on day 0 (A), 1 (B), 2 (C), 7 (D), 10 (E), and 14 (F) and control limbs on day $0(\mathrm{G}), 1(\mathrm{H}), 2(\mathrm{I}), 7(\mathrm{~J}), 10(\mathrm{~K})$, and $14(\mathrm{~L})$. Arrowheads indicate the infiltrating mononuclear inflammatory cells in the ischemic limbs. Scale bar $=100 \mu \mathrm{m}$.

YB-1 regulates the transcription of various genes in response to cellular stress such as drug toxicity, reactive oxygen, UV damage, and low temperature (Kohno et al. 2003; Fujita et al. 2005; Hyogotani et al. 2012; Ito et al. 2012). In addition, several genes important for wound healing and cellular proliferation and survival are regulated by YB-1, including collagen alpha1 and alpha2 (Norman et al. 2001; Higashi et al. 2003), matrix metalloproteinase 2 (Mertens et al. 1997), the B-chain isoform of plateletderived growth factor (Stenina et al. 2000), vascular endothelial growth factor (Coles et al. 2004), granulocyte-macrophage-colony stimulating factor (Coles et al. 2000), and Fas death receptor (Lasham et al. 2000); moreover, YB-1 can also regulate $\alpha \mathrm{SMA}$ and human pulmonary myofibro- blasts (Zhang et al. 2005). Takahashi et al. (2010) reported that YB-1 is increased in the angiogenic endothelial cells of various tumors and is involved in the growth of endothelial cells. These previous findings suggest the possibility that YB-1 is involved in tissue regeneration in various situations in vivo, including tissue regeneration after ischemic injury.

Miwa et al. (2006) analyzed YB-1 expression in various tissues at different stages of the mouse life cycle and reported that its expression is regulated with growth or aging in a tissue-specific manner and that it is markedly expressed at the prenatal stage, and then completely downregulated suddenly in the skeletal muscles after birth. In the present study, YB-1 localized to the regenerated myotubes that expressed MyoD from 7 days after ischemic 
A

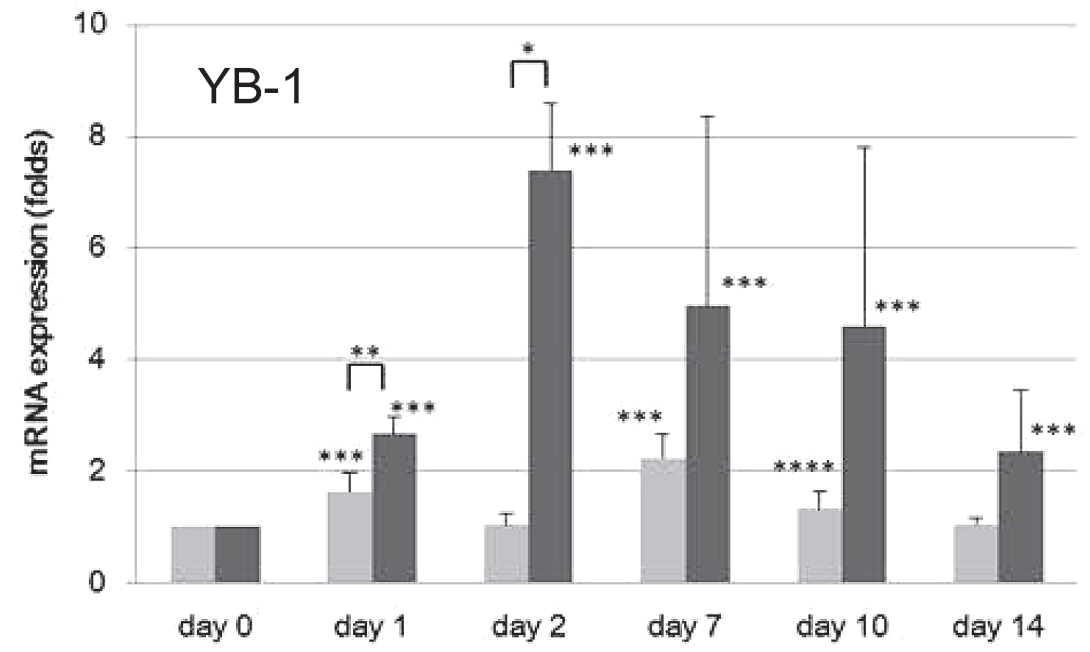

B

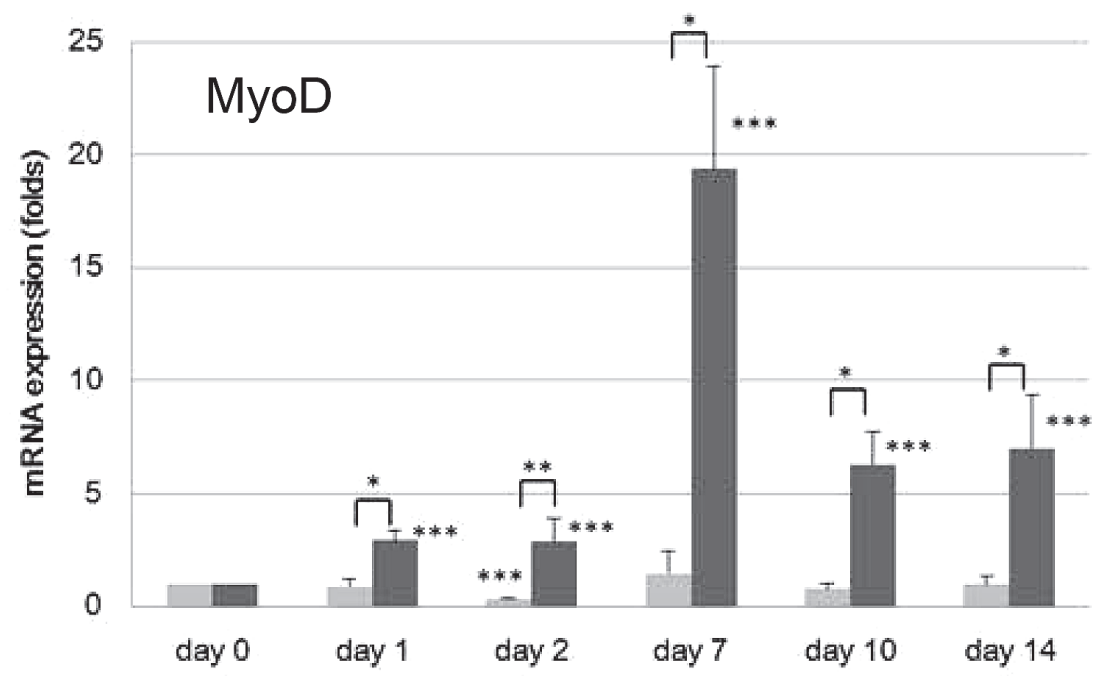

C

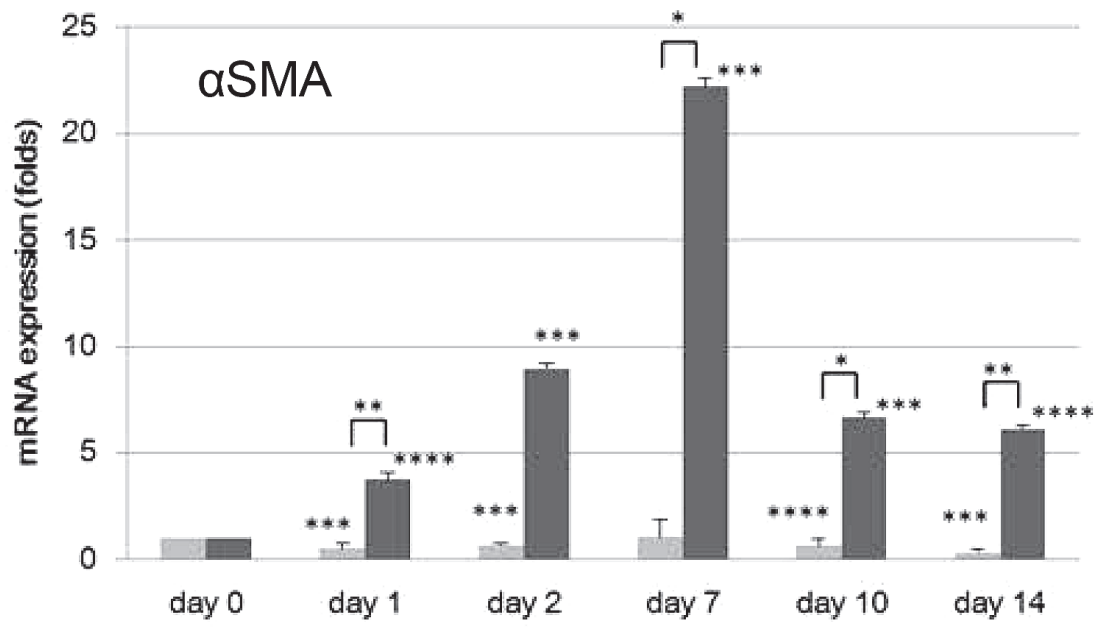

control limbs a ischemic limbs

Fig. 3. Time-dependent changes in YB-1, MyoD, and $\alpha$ SMA mRNA levels in ischemic limb muscles.

Relative mRNA levels of YB-1 (A), MyoD (B), and $\alpha$ SMA (C) in the ischemic limb muscles (dark bars) and the control limb muscles (gray bars) from day 0 to day 14 were determined by RT-PCR analysis. Significant differences in mRNA levels between the ischemic limbs and the control limbs on the same day are indicated as $*(p<0.01)$ and $* *(p<0.05)$. Significant differences in mRNA levels compared with those in the corresponding limbs on day 0 are indicated as $* * *(p<0.01)$ and $* * * *(p<0.05)$. 
YB-1
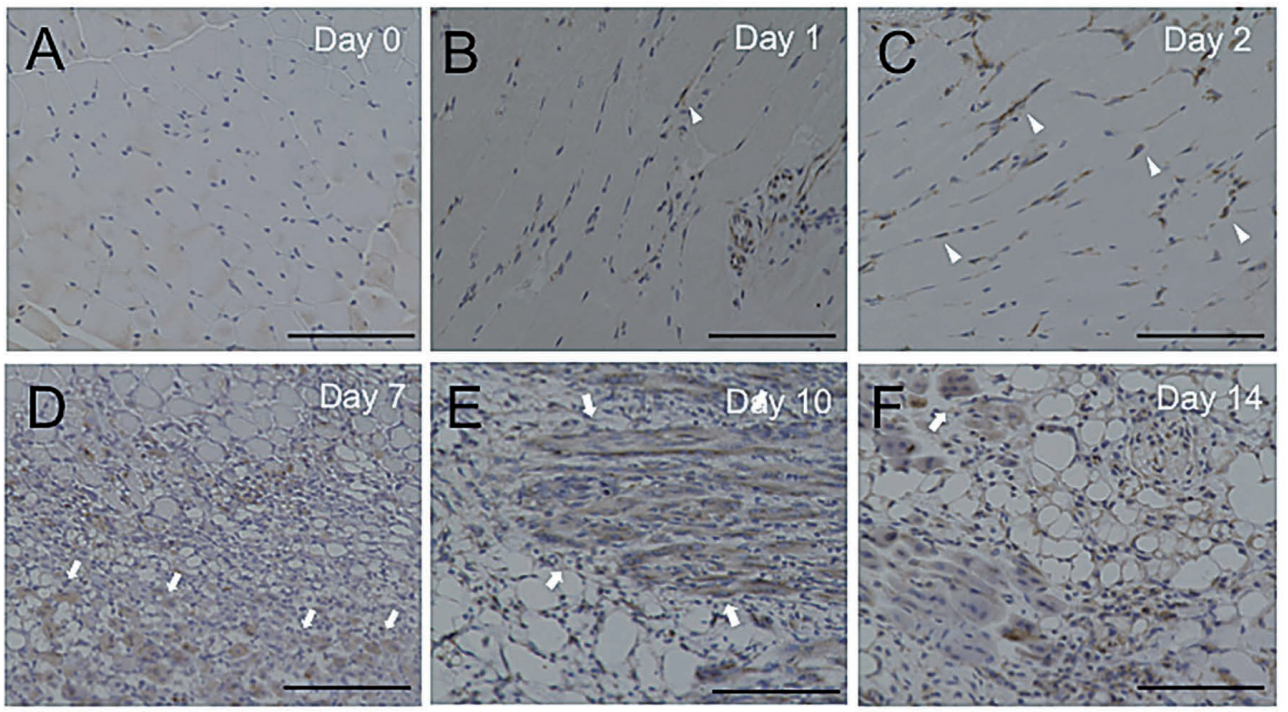

\section{CD31}

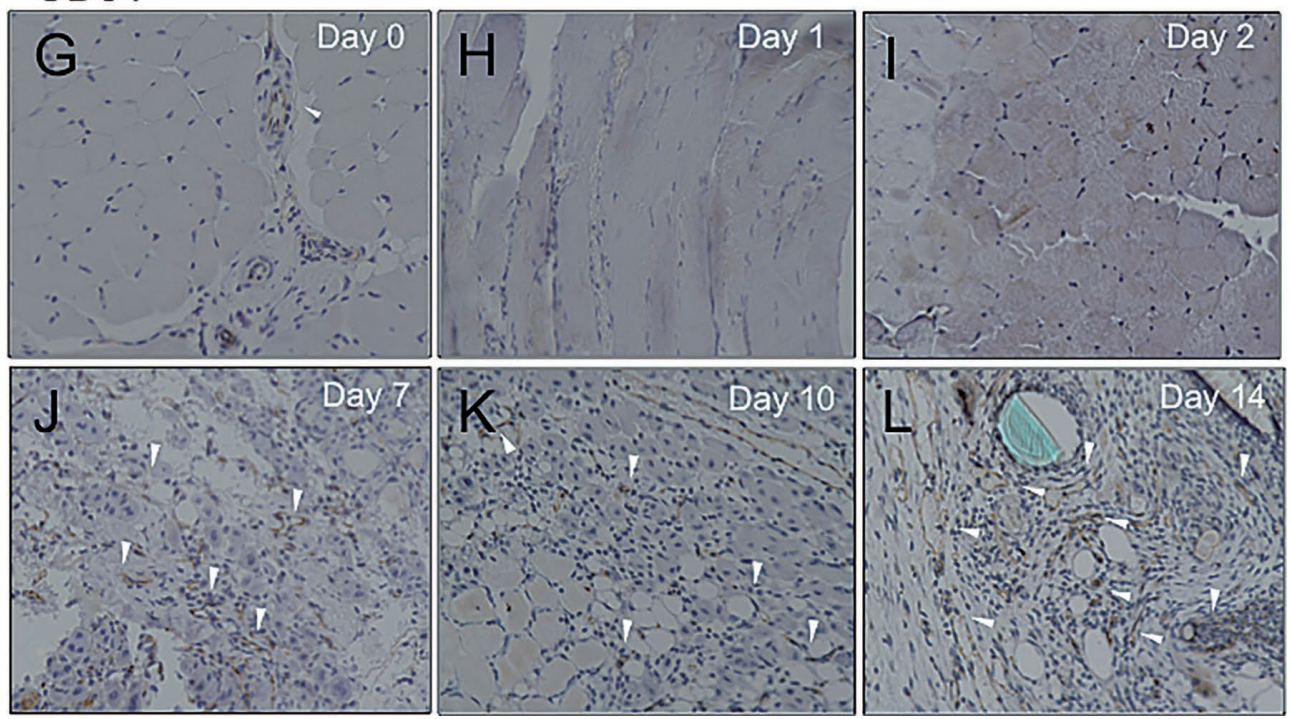

Fig. 4. Immunohistochemical staining for YB-1 and CD31 in ischemic limbs.

Immunohistochemical staining for YB-1 in the ischemic hind limbs on day 0 (A), 1 (B), 2 (C), 7 (D), 10 (E), and 14 (F). Arrowheads in (B) and (C) indicate the mononuclear inflammatory cells. Arrows in (D), (E), and (F) indicate myoblasts and myotubes. Immunohistochemical staining for CD31 in the ischemic hind limbs on day $0(\mathrm{G}), 1(\mathrm{H}), 2$ (I), 7 (J), 10 $(\mathrm{K})$, and $14(\mathrm{~L})$. Arrowheads in $(\mathrm{G}),(\mathrm{J}),(\mathrm{K})$, and (L) indicate endothelial cells. Scale bar $=100 \mu \mathrm{m}$.

injury. The present findings, along those of Miwa et al. (2006), suggested the possibility that YB-1 is involved in the maturation of muscle from myoblasts.

aSMA is one of the few genes for which expression is relatively restricted to vascular smooth muscle cells. $\alpha \mathrm{SMA}$ is a transient component of stress fibers, which are structures that play an important role in cell adhesion and tension. $\alpha$ SMA associates with stress fibers to increase cell traction force under specific physiological conditions, and it has also been reported to be expressed in myofibroblasts and myoepithelial cells. SMemb is also a marker of proliferating embryonic smooth muscle cells. Notably, both $\alpha \mathrm{SMA}$ and SMemb were transiently increased in the myo- tubes of regenerated skeletal muscles after ischemic injury in our model. Lu et al. (2011) reported the successful differentiation of muscle-derived cells, isolated from human skeletal muscles, into smooth muscle cells. Thus, our findings together with those of the previous study suggest the potential of myoblasts in skeletal muscle tissues to differentiate into smooth muscle cells.

In contrast, David et al. (2012) reported that YB-1 is involved in the de-differentiation of cardiomyocytes after transplantation by upregulating $\alpha \mathrm{SMA}$ in a murine heterotopic heart graft model. In addition, they reported that the phosphorylated form of YB-1 is expressed in the human ventricular cardiomyocytes of transplanted hearts obtained 
MyoD
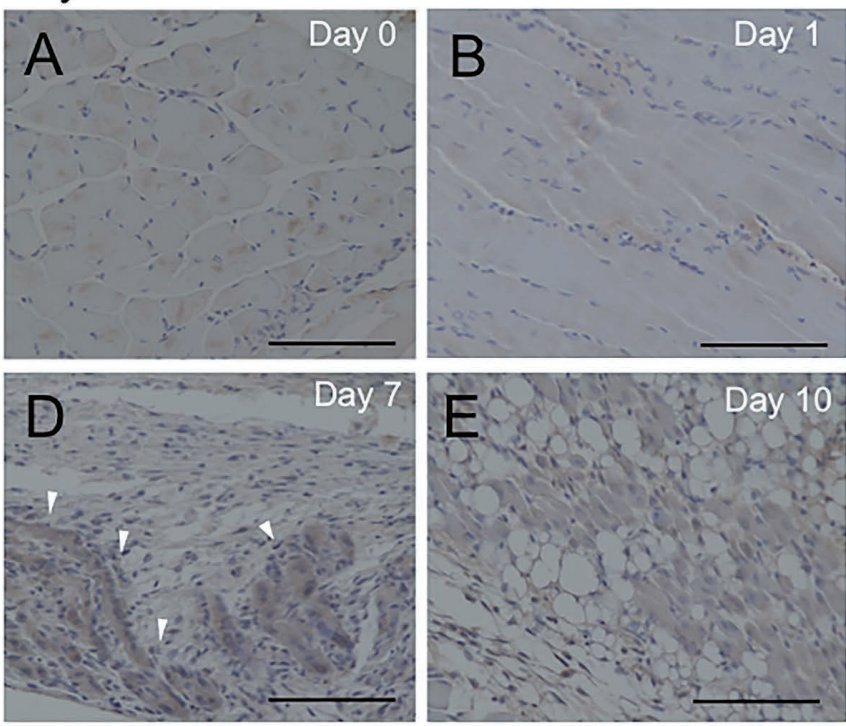

$\alpha$ SMA
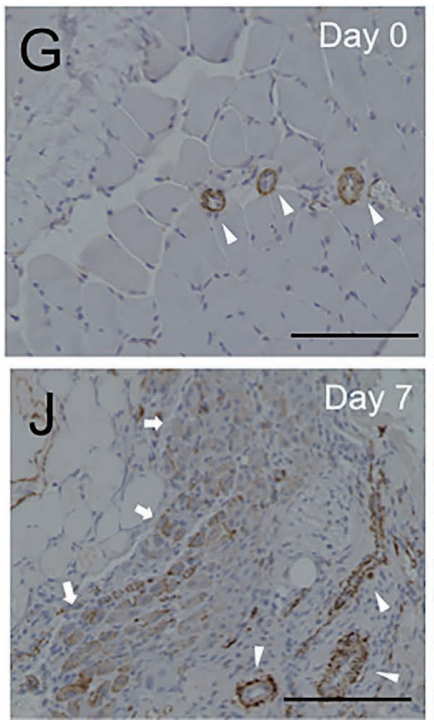
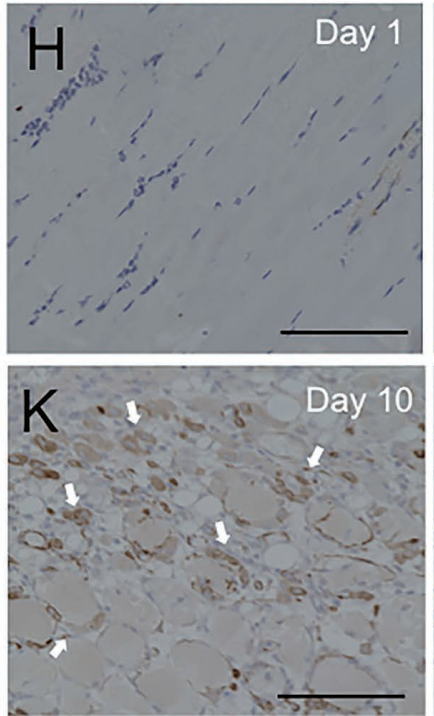
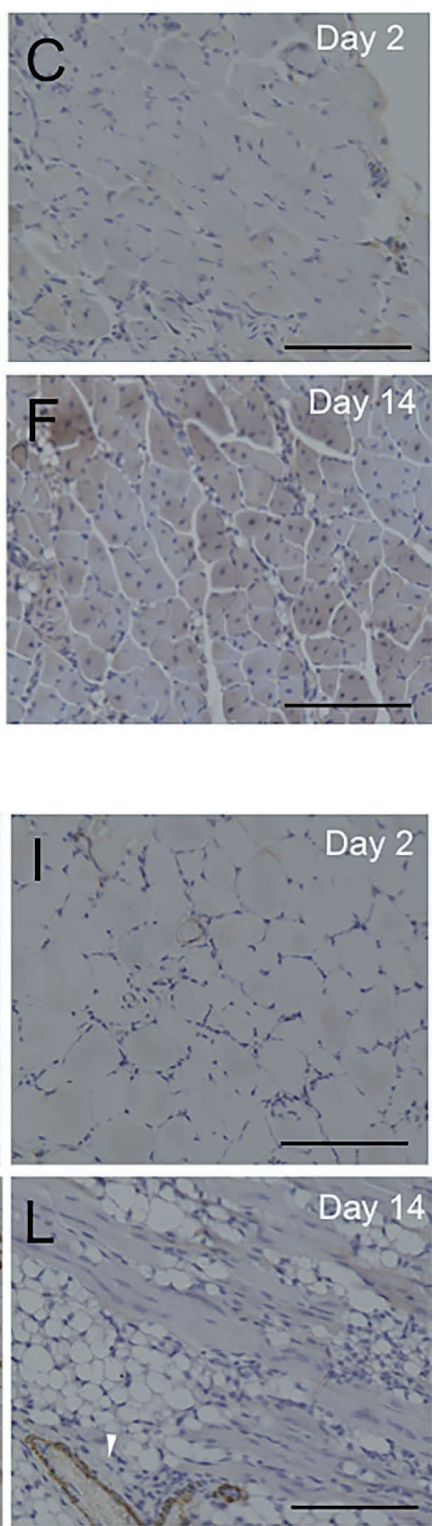

Fig. 5. Immunohistochemical staining for MyoD and $\alpha \mathrm{SMA}$ in ischemic limbs.

Immunohistochemical staining for MyoD in ischemic hind limbs on day 0 (A), 1 (B), 2 (C), 7 (D), 10 (E), and 14 (F). Arrowheads in (D) indicate newly generated myoblasts and myotubes. Immunohistochemical staining for $\alpha \mathrm{SMA}$ in the ischemic hind limbs on day $0(\mathrm{G}), 1(\mathrm{H}), 2(\mathrm{I}), 7(\mathrm{~J}), 10(\mathrm{~K})$, and $14(\mathrm{~L})$. Arrowheads in $(\mathrm{G}),(\mathrm{J})$, and $(\mathrm{L})$ indicate vascular smooth muscle cells. Arrows in $(\mathrm{J})$ and $(\mathrm{K})$ indicate the myotubes in the regenerated muscles. Scale bar $=100 \mu \mathrm{m}$.

after endomyocardial biopsy, indicating the indispensable role of YB-1 in the remodeling of cardiac tissue in transplanted hearts (David et al. 2012). $\alpha$ SMA-expressing myofibroblasts have been detected in the stroma of non-malignant tissues during wound repair, and the dysregulation of $\alpha$ SMA-positive myofibroblasts has been associated with various fibrotic diseases including atherosclerosis. $\alpha \mathrm{SMA}$ is expressed in various myogenic soft tissue tumors including leiomyomas and leiomyosarcomas, among others. Thus, it is believed that $\alpha \mathrm{SMA}$ is essential for the normal differentiation and function of myofibroblasts. The present findings, together with those of David et al. (2012), suggest that YB-1 is an important factor involved in the regulation of $\alpha \mathrm{SMA}$.

MyoD belongs to the family of myogenic regulatory factors and plays a key role in the regulation of muscle differentiation. MyoD has also been reported to play a role in the regulation of muscle repair (Berkes and Tapscott 2005). In the present study, MyoD was localized to the myosatellite cells and myoblasts during the acute phase of ischemic injury, and its levels in the newly generated myotubes peaked on day 7. Moreover, MyoD levels decreased drastically on day 10 , which was accompanied by a gradual reduction in YB-1 levels in the ischemic limbs. Conversely, 

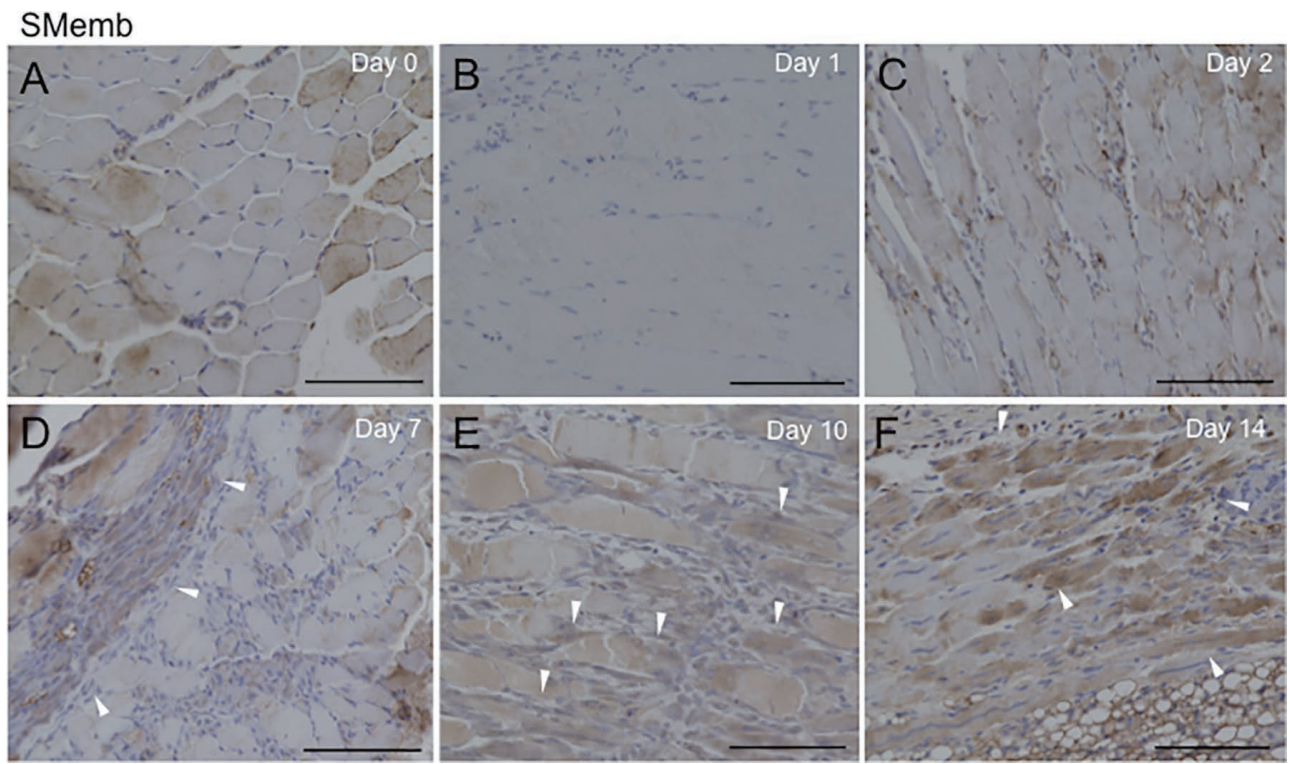

Fig. 6. Immunohistochemical staining for SMemb in ischemic limbs.

Immunohistochemical staining for SMemb in ischemic hind limbs on day 0 (A), 1 (B), 2 (C), 7 (D), 10 (E), and 14 (F).

Arrowheads indicate the regenerated myotubes in the ischemic limbs. Scale bar $=100 \mu \mathrm{m}$.
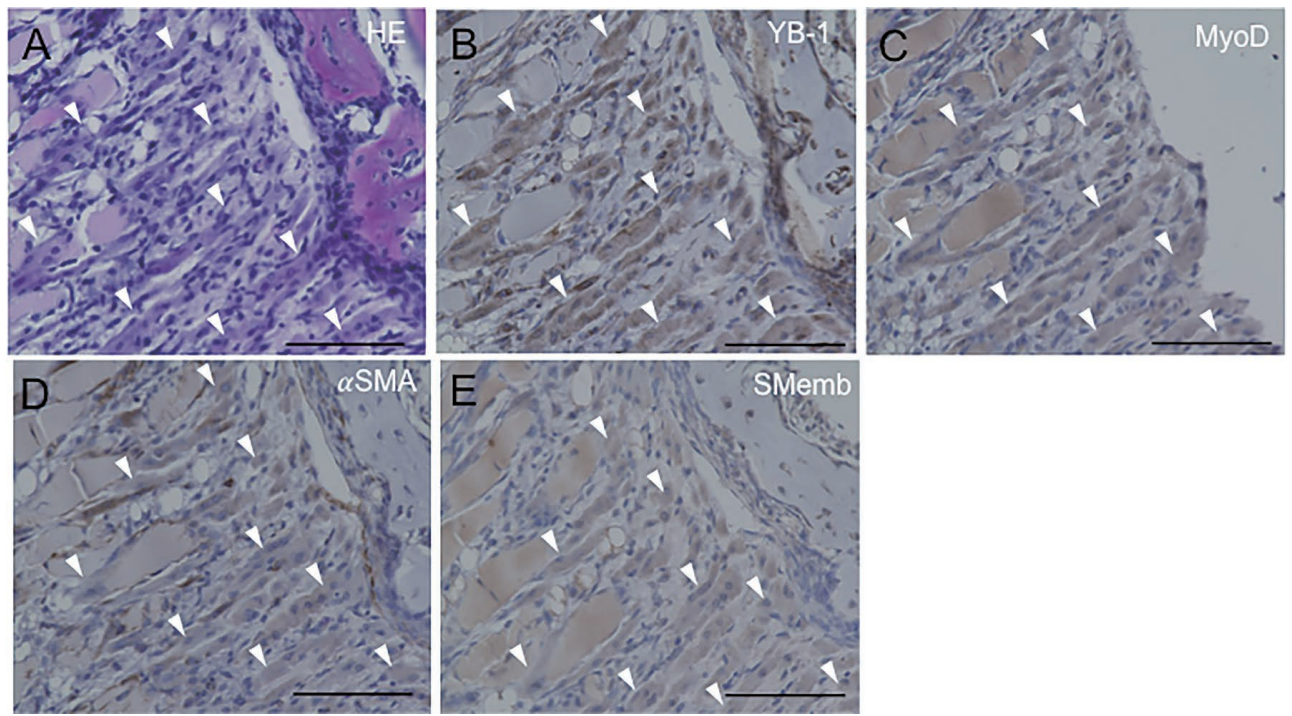

Fig. 7. Histopathological examination of ischemic limbs on day 10.

(A) Hematoxylin and eosin staining, (B-E) immunohistochemical staining for YB-1 (B), MyoD (C), $\alpha$ SMA (D), and SMemb (E) in ischemic hind limbs on day 10. Arrowheads indicate the regenerated myotubes in the ischemic limbs. Scale bar $=100 \mu \mathrm{m}$.

Song and Lee (2010) reported a novel function for YB-1 as a regulator of skeletal muscle differentiation. They reported that YB-1 binds to the $M y o D$ core enhancer region and negatively regulates $M y o D$ transcription, which in turn prevents $\mathrm{C} 2 \mathrm{C} 12$ myoblast cells from undergoing terminal differentiation through interactions with cell type-specific regulators such as Msx1 homeoprotein (Song and Lee 2010). Hence, long-term expression of YB-1 might terminate the MyoDmediated differentiation of myocytes after forming myotubes in ischemic limbs.

Recently, the YB-1 was reported to directly regulate hypoxia-inducible factor (HIF) $1 \alpha$, a critical transcription factor that regulates adaptive responses to hypoxia and plays a pivotal role in wound healing (El-Naggar et al. 2015; Xu et al. 2017). Although the association between YB-1 and HIF $1 \alpha$ was not investigated in the present study, it is possible that the increased expression of YB-1 after ischemic injury could enhance wound healing via increased expression of HIF $1 \alpha$.

In conclusion, to our knowledge, the present study is the first to report the increase in YB-1 expression in ischemic limbs, which was associated with alterations in the 
expression of several molecules that are important for muscle regeneration. Although further studies are required to elucidate the direct interaction between YB-1 and these myogenic molecules, the present study, together with previous studies, suggests an indispensable role for YB-1 in the regeneration of muscles in various situations.

\section{Acknowledgments}

The authors thank Ms. Shinobu Kamijo for her excellent technical assistance.

\section{Conflict of Interest}

The authors declare no conflict of interest.

\section{References}

Belch, J.J., Topol, E.J., Agnelli, G., Bertrand, M., Califf, R.M., Clement, D.L., Creager, M.A., Easton, J.D., Gavin, J.R. 3rd, Greenland, P., Hankey, G., Hanrath, P., Hirsch, A.T., Meyer, J., Smith, S.C., et al. (2003) Critical issues in peripheral arterial disease detection and management: a call to action. Arch. Intern. Med., 163, 884-892.

Berkes, C.A. \& Tapscott, S.J. (2005) MyoD and the transcriptional control of myogenesis. Semin. Cell Dev. Biol., 16, 585-595.

Coles, L.S., Bartley, M.A., Bert, A., Hunter, J., Polyak, S., Diamond, P., Vadas, M.A. \& Goodall, G.J. (2004) A multiprotein complex containing cold shock domain (Y-box) and polypyrimidine tract binding proteins forms on the vascular endothelial growth factor mRNA. Potential role in mRNA stabilization. Eur. J. Biochem., 271, 648-660.

Coles, L.S., Diamond, P., Occhiodoro, F., Vadas, M.A. \& Shannon, M.F. (2000) An ordered array of cold shock domain repressor elements across tumor necrosis factor-responsive elements of the granulocyte-macrophage colony-stimulating factor promoter. J. Biol. Chem. 275, 14482-14493.

David, J.J., Subramanian, S.V., Zhang, A., Willis, W.L., Kelm, R.J. Jr., Leier, C.V. \& Strauch, A.R. (2012) Y-box binding protein-1 implicated in translational control of fetal myocardial gene expression after cardiac transplant. Exp. Biol. Med., 237, 593-607.

Didier, D.K., Schiffenbauer, J., Woulfe, S.L., Zacheis, M. \& Schwartz, B.D. (1988) Characterization of the cDNA encoding a protein binding to the major histocompatibility complex class II Y box. Proc. Natl. Acad. Sci. USA, 85, 7322-7326.

El-Naggar, A.M., Veinotte, C.J., Cheng, H., Grunewald, T.G., Negri, G.L., Somasekharan, S.P., Corkery, D.P., Tirode, F., Mathers, J., Khan, D., Kyle, A.H., Baker, J.H., LePard, N.E., McKinney, S., Hajee, S., et al. (2015) Translational activation of HIF1 1 aby YB-1 promotes sarcoma metastasis. Cancer Cell, 27, 682-697.

Fujita, T., Ito, K., Izumi, H., Kimura, M., Sano, M., Nakagomi, H., Maeno, K., Hama, Y., Shingu, K., Tsuchiya, S., Kohno, K. \& Fujimori, M. (2005) Increased nuclear localization of transcription factor Y-box binding protein 1 accompanied by up-regulation of P-glycoprotein in breast cancer pretreated with paclitaxel. Clin. Cancer Res., 11, 8837-8844.

Furukawa, M., Uchiumi, T., Nomoto, M., Takano, H., Morimoto, R.I., Naito, S., Kuwano, M. \& Kohno, K. (1998) The role of an inverted CCAAT element in transcriptional activation of the human DNA topoisomerase II $\alpha$ gene by heat shock. $J$. Biol. Chem., 273, 10550-10555.

Higashi, K., Inagaki, Y., Fujimori, K., Nakao, A., Kaneko, H. \& Nakatsuka, I. (2003) Interferon- $\gamma$ interferes with transforming growth factor- $\beta$ signaling through direct interaction of YB-1 with Smad3. J. Biol. Chem., 278, 43470-43479.

Hyogotani, A., Ito, K., Yoshida, K., Izumi, H., Kohno, K. \&
Amano, J. (2012) Association of nuclear YB-1 localization with lung resistance-related protein and epidermal growth factor receptor expression in lung cancer. Clin. Lung Cancer, 13, 375-384.

Ito, T., Kamijo, S., Izumi, H., Kohno, K., Amano, J. \& Ito, K. (2012) Alteration of Y-box binding protein-1 expression modifies the response to endocrine therapy in estrogen receptor-positive breast cancer. Breast Cancer Res. Treat., 133, 145-159.

Kamalov, G., Varma, B.R., Lu, L., Sun, Y., Weber, K.T. \& Guntaka, R.V. (2005) Expression of the multifunctional Y-box protein, YB-1, in myofibroblasts of the infarcted rat heart. Biochem. Biophys. Res. Commun., 334, 239-244.

Kawamoto, A., Katayama, M., Handa, N., Kinoshita, M., Takano, H., Horii, M., Sadamoto, K., Yokoyama, A., Yamanaka, T., Onodera, R., Kuroda, A., Baba, R., Kaneko, Y., Tsukie, T., Kurimoto, Y., et al. (2009) Intramuscular transplantation of G-CSF-mobilized CD34(+) cells in patients with critical limb ischemia: a phase I/IIa, multicenter, single-blinded, doseescalation clinical trial. Stem cells., 27, 2857-2864.

Kohno, K., Izumi, H., Uchiumi, T., Ashizuka, M. \& Kuwano, M. (2003) The pleiotropic functions of the Y-box-binding protein, YB-1. Bioessays, 25, 691-698.

Lasham, A., Lindridge, E., Rudert, F., Onrust, R. \& Watson, J. (2000) Regulation of the human fas promoter by YB-1, Purd and AP-1 transcription factors. Gene, 252, 1-13.

Lu, S.H., Lin, A.T., Chen, K.K., Chiang, H.S. \& Chang, L.S. (2011) Characterization of smooth muscle differentiation of purified human skeletal muscle-derived cells. J. Cell. Mol. Med., 15, 587-592.

McDermott, M.M., Kerwin, D.R., Liu, K., Martin, G.J., O'Brien, E., Kaplan, H. \& Greenland, P. (2001) Prevalence and significance of unrecognized lower extremity peripheral arterial disease in general medicine practice. J. Gen. Intern. Med., 16, 384-390.

Mertens, P.R., Harendza, S., Pollock, A.S. \& Lovett, D.H. (1997) Glomerular mesangial cell-specific transactivation of matrix metalloproteinase 2 transcription is mediated by YB-1. $J$. Biol. Chem., 272, 22905-22912.

Miwa, A., Higuchi, T. \& Kobayashi, S. (2006) Expression and polysome association of YB-1 in various tissues at different stages in the lifespan of mice. Biochim. Biophys. Acta, 1760, 1675-1681.

Norgren, L., Hiatt, W.R., Dormandy, J.A., Nehler, M.R., Harris, K.A. \& Fowkes, F.G.; TASK II Working Group (2007) InterSociety consensus for the management of peripheral arterial disease (TASC II). J. Vasc. Surg., 45 Suppl S, S5-67.

Norman, J.T., Lindahl, G.E., Shakib, K., En-Nia, A., Yilmaz, E. \& Mertens, P.R. (2001) The Y-box binding protein YB-1 suppresses collagen $\alpha 1$ (I) gene transcription via an evolutionarily conserved regulatory element in the proximal promoter. J. Biol. Chem., 276, 29880-29890.

Rowlands, T.E. \& Donnelly, R. (2007) Medical therapy for intermittent claudication. Eur. J. Vasc. Endovasc. Surg., 34, 314-321.

Song, Y.J. \& Lee, H. (2010) YB1/p32, a nuclear Y-box binding protein 1 , is a novel regulator of myoblast differentiation that interacts with Msx1 homeoprotein. Exp. Cell Res., 316, 517-529.

Stenina, O.I., Poptic, E.J. \& DiCorleto, P.E. (2000) Thrombin activates a $\mathrm{Y}$ box-binding protein (DNA-binding protein $\mathrm{B}$ ) in endothelial cells. J. Clin. Invest., 106, 579-587.

Suzuki, H. \& Iso, Y. (2013) Clinical application of vascular regenerative therapy for peripheral artery disease. Biomed. Res. Int., 2013, doi.org/10.1155/2013/179730.

Takahashi, M., Shimajiri, S., Izumi, H., Hirano, G., Kashiwagi, E., Yasuniwa, Y., Wu, Y., Han, B., Akiyama, M., Nishizawa, S., Sasaguri, Y. \& Kohno, K. (2010) Y-box binding protein-1 is a novel molecular target for tumor vessels. Cancer Sci., 101, 
1367-1373.

Weitz, J.I., Byrne, J., Clagett, G.P., Farkouh, M.E., Porter, J.M., Sackett, D.L., Strandness, D.E. Jr. \& Taylor, L.M. (1996) Diagnosis and treatment of chronic arterial insufficiency of the lower extremities: a critical review. Circulation, 94, 30263049.

Wolffe, A.P., Tafuri, S., Ranjan, M. \& Familari, M. (1992) The Y-box factors: a family of nucleic acid binding proteins conserved from Escherichia coli to man. New Biol., 4, 290-298.
Xu, L., Li, H., Wu, L. \& Huang, S. (2017) YBX1 promotes tumor growth by elevating glycolysis in human bladder cancer. Oncotarget, 8, 65946-65956.

Zhang, A., Liu, X., Cogan, J.G., Fuerst, M.D., Polikandriotis, J.A., Kelm, R.J. Jr. \& Strauch, A.R. (2005) YB-1 coordinates vascular smooth muscle $\alpha$-actin gene activation by transforming growth factor $\beta 1$ and thrombin during differentiation of human pulmonary myofibroblasts. Mol. Biol. Cell, 16, 4931-4940. 